\title{
Heavier- and lighter-load isolated lumbar extension resistance training produce similar strength increases, but different perceptual responses, in healthy males and females
}

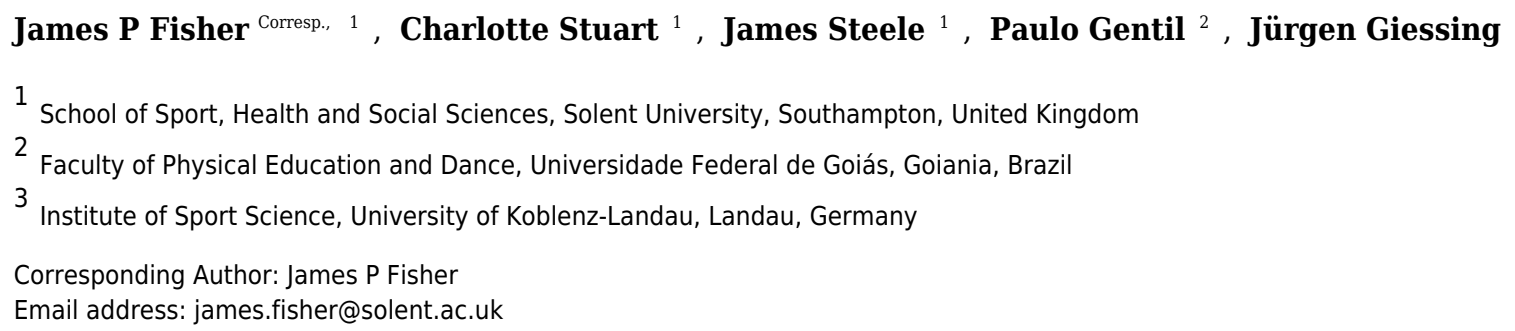

Objectives: Muscles dominant in type I muscle fibres, such as the lumbar extensors, are often trained using lighter loads and higher repetition ranges. However, literature suggests that similar strength adaptations can be attained by the use of both heavier- $(\mathrm{HL})$ and lighter-load (LL) resistance training across a number of appendicular muscle groups. Furthermore, LL resistance exercise to momentary failure might result in greater discomfort. Design: The aims of the present study were to compare strength adaptations, as well as perceptual responses of effort (RPE-E) and discomfort (RPE-D), to isolated lumbar extension (ILEX) exercise using HL ( $80 \%$ of maximum voluntary contraction; MVC) and LL ( $50 \%$ MVC) in healthy males and females. Methods: Twenty-six participants $(n=14$ males, $n=12$ females) were divided in to sex counter-balanced HL ( $23 \pm 5$ years; 172.3 $\pm 9.8 \mathrm{~cm} ; 71.0 \pm 13.1 \mathrm{~kg}$ ) and $\mathrm{LL}(22 \pm 2$ years; $175.3 \pm 6.3 \mathrm{~cm} ; 72.8 \pm 9.5 \mathrm{~kg}$ ) resistance training groups. All participants performed a single set of dynamic ILEX exercise 1 day/week for 6 weeks using either $80 \%$ (HL) or $50 \%$ (LL) of their MVC to momentary failure. Results: Analyses revealed significant pre- to post-intervention increases in isometric strength for both $\mathrm{HL}$ and $\mathrm{LL}$, with no significant between-group differences ( $p>$ 0.05). Changes in strength index (area under torque curves) were $2891 \mathrm{Nm}$.degrees [95\%Cls 1612 to 4169] and $2865 \mathrm{Nm}$-degrees [95\%Cls 1587 to 4144] for HL and LL respectively. Changes in MVC were $51.7 \mathrm{Nm}$ [95\%Cls 24.4 to 79.1 ] and $46.0 \mathrm{Nm}$ [95\%Cls 18.6 to 73.3] for HL and LL respectively. Mean repetitions per set, total training time and discomfort were all significantly higher for LL compared to HL ( $26 \pm 8$ vs. $8 \pm 3$ repetitions, $158.5 \pm 47$ vs. $50.5 \pm 15$ seconds, and $7.8 \pm 1.8$ vs. $4.8 \pm 2.5$, respectively; all $p<0.005$ ).

Conclusions: The present study supports that that low-volume, low-frequency ILEX resistance exercise can produce similar strength increases in the lumbar extensors using either HL or LL. As such personal trainers, trainees and strength coaches can consider 
other factors which might impact acute performance (e.g. effort and discomfort during the exercise). This data might prove beneficial in helping asymptomatic persons reduce the risk of low-back pain, and further research, might consider the use of HL exercise for chronic low-back pain symptomatic persons. 
1 Heavier- and lighter-load isolated lumbar extension resistance training produce similar strength

2 increases, but different perceptual responses, in healthy males and females

3

4 Running Title: Heavier- and Lighter-Load isolated lumbar extension exercise

5

6 Original research article

7

8 James P Fisher ${ }^{1}$, Charlotte Stuart ${ }^{1}$, James Steele ${ }^{1}$, Paulo Gentil ${ }^{2}$, Jürgen Giessing ${ }^{3}$

9

10

${ }^{1}$ Southampton Solent University, East Park Terrace, Southampton, UK

11

${ }^{2}$ Faculty of Physical Education and Dance, Federal University of Goias, Goiania, Brazil

12

${ }^{3}$ Institute of Sport Science, University of Koblenz-Landau, Landau, Germany

13

14

Corresponding Author: James Fisher, Southampton Solent University, East Park Terrace, Southampton,

UK. Tel: +44 2382013163 Email: james.fisher@solent.ac.uk

Co-Author contact details:

Charlotte Stuart (charlotte.stuart95@outlook.com)

James Steele (James.Steele@solent.ac.uk)

Paulo Gentil (paulogentil@hotmail.com)

Jürgen Giessing (giessing@uni-landau.de)

22

23

24

Word count: 3551

25 


\section{Abstract}

Objectives: Muscles dominant in type I muscle fibres, such as the lumbar extensors, are often trained using lighter loads and higher repetition ranges. However, literature suggests that similar strength adaptations can be attained by the use of both heavier- $(\mathrm{HL})$ and lighter-load (LL) resistance training across a number of appendicular muscle groups. Furthermore, LL resistance exercise to momentary failure might result in greater discomfort. Design: The aims of the present study were to compare strength adaptations, as well as perceptual responses of effort (RPE-E) and discomfort (RPE-D), to isolated lumbar extension (ILEX) exercise using $\mathrm{HL}$ ( $80 \%$ of maximum voluntary contraction; MVC) and LL (50\% MVC) in healthy males and females. Methods: Twenty-six participants ( $n=14$ males, $n=12$ females) were divided in to sex counter-balanced HL ( $23 \pm 5$ years; $172.3 \pm 9.8 \mathrm{~cm} ; 71.0 \pm 13.1 \mathrm{~kg}$ ) and LL (22 \pm 2 years; $175.3 \pm 6.3 \mathrm{~cm} ; 72.8 \pm 9.5 \mathrm{~kg}$ ) resistance training groups. All participants performed a single set of dynamic ILEX exercise 1 day/week for 6 weeks using either $80 \%$ (HL) or $50 \%$ (LL) of their MVC to momentary failure. Results: Analyses revealed significant pre- to post-intervention increases in isometric strength for both HL and LL, with no significant between-group differences $(p>0.05)$. Changes in strength index (area under torque curves) were $2891 \mathrm{Nm} \cdot$ degrees [95\%Cls 1612 to 4169] and $2865 \mathrm{Nm} \cdot$ degrees [95\%Cls 1587 to 4144 ] for HL and LL respectively. Changes in MVC were $51.7 \mathrm{Nm}$ [95\%Cls 24.4 to 79.1] and 46.0 $\mathrm{Nm}$ [95\%Cls 18.6 to 73.3$]$ for $\mathrm{HL}$ and LL respectively. Mean repetitions per set, total training time and discomfort were all significantly higher for LL compared to HL ( $26 \pm 8$ vs. $8 \pm 3$ repetitions, $158.5 \pm 47$ vs. $50.5 \pm 15$ seconds, and $7.8 \pm 1.8$ vs. $4.8 \pm 2.5$, respectively; all $p<0.005)$. Conclusions: The present study supports that that low-volume, low-frequency ILEX resistance exercise can produce similar strength increases in the lumbar extensors using either HL or LL. As such personal trainers, trainees and strength coaches can consider other factors which might impact acute performance (e.g. effort and discomfort during the exercise). This data might prove beneficial in helping asymptomatic persons reduce the risk of 
51 low-back pain, and further research, might consider the use of HL exercise for chronic low-back pain

52 symptomatic persons.

53

54

55

\section{INTRODUCTION}

Resistance training (RT) to momentary failure (MF), defined as "the set end-point when trainees reach the point where despite attempting to do so they cannot complete the concentric portion of their current repetition", 1 appears to produce similar strength adaptations when using both heavier- (HL; $>60 \%$ 1-repetition maximum; 1RM) and lighter-loads (LL; $<60 \% 1 \mathrm{RM}$ ) particularly when tested by impartial means (e.g. isometric testing following dynamic training). ${ }^{2,3,4}$ This is argued to potentially result from maximal synchronous, or sequential, motor unit recruitment for $\mathrm{HL}$ and $\mathrm{LL}$ respectively, ${ }^{2}$ as per the size principle. ${ }^{5,6}$ Furthermore, it has been proposed that complete motor unit recruitment may be a driving factor towards optimizing strength and hypertrophic adaptations. ${ }^{2,7}$

A growing area of research considers the disparity between effort and its perception; "the amount of mental or physical energy being given to a task" which is determined by the current ability to meet task demands relative to those demands; and discomfort "the physiological and unpleasant sensations associated with exercise" 8,9 . A practical example of this is that "A short maximal voluntary contraction for leg extension, for example, will by nature induce a maximal sense of effort while, initially, other unpleasant sensations will probably be modest. Repeating this maximal contraction several times, however, will increase these unpleasant sensations continuously, whereas the sense of effort will be always the same (i.e. maximal)."10

Fisher, et al. ${ }^{2}$ recently hypothesised that disparity in adaptations following RT at HL or LL might be a result of persons anchoring their perceptions of effort upon their perceptions of discomfort. For example, unless specifically instructed to differentiate the two, most trainees evidently conflate them instead anchoring their perceptions of effort upon their perceptions of discomfort. ${ }^{11}$ Indeed, it has been 
74

argued that this can result in trainees underestimating their proximity to $\mathrm{MF}^{12,13}$ and this effect may be exacerbated under low load conditions. ${ }^{14,15} \mathrm{~A}$ number of studies where participants have been instructed to differentiate their perceptions of effort and discomfort ${ }^{11}$ have reported greater discomfort for more fatiguing conditions using lower loads, higher time under load and/or repetitions performed. ${ }^{3,16,17}$ It seems likely that this results from afferent feedback ${ }^{9}$ due to a decrease in $\mathrm{pH}$, elevated blood lactate (BLa), cortisol, and inorganic phosphate $\left(\mathrm{P}_{\mathrm{i}}\right)$ along with increases in $\mathrm{H}^{+}$as a result of the prolonged elevated adenosine triphosphate (ATP) production. ${ }^{18-21}$

Perceptions of discomfort appear to be linked to afferent feedback and thus a reason why increased metabolic stress may promote greater discomfort during LL resistance exercise. ${ }^{9}$

Contrastingly, perceptions of effort are likely related to central motor output to drive motor unit recruitment ${ }^{22}$ which may explain similar perceptions of maximal effort when both $L L$ and $H L$ are performed to MF. Considering the potential role of motor unit recruitment in determining adaptation to $\mathrm{RT}$ it is important to understand the interactions between load, effort, and discomfort. Within the muscles of the trunk and spine there is a predominance of type I muscle fibres ${ }^{23}$ to sustain the repeated lower force actions needed for postural control and stability. As such, a historical approach has been to train these muscles using LL for a greater number of repetitions.

An area which has received little consideration with respect to the comparison of $H L$ and $L L$ is that of isolated lumbar extension (ILEX) exercise. Low-volume (e.g. single-set) and low-frequency (1 day/week) ILEX exercise is shown to produce considerable strength increases both in trained persons ${ }^{24,25}$ and those symptomatic of chronic low-back pain. ${ }^{26-28}$ However, previous studies ${ }^{29,30}$ looking to compare different loading schemes using ILEX RT have used similarly low loads in both HL ( $35 \%$ and $50 \%$ of MVC) and LL conditions ( $20 \%$ of MVC). Furthermore, participants did not train to MF, which has been

\footnotetext{
${ }^{1}$ Using a 0-10 scale for effort and discomfort these have been anchored as $0=$ no exertion and 10=maximal effort, and $0=$ no discomfort and $10=$ maximal discomfort, respectively.
} 
96

97

98

99

100

101

102

103

104

105

106

107

108

109

110

111

112

114

115

116

117

suggested as necessary to standardize RT interventions ${ }^{31}$. Indeed, it has been noted that there is a lack of research considering different loading patterns for ILEX $\mathrm{RT}^{32}$, and since the lumbar extensors have been identified as a muscle group that appears to require isolated (e.g. single-joint) exercise to sufficiently strengthen them ${ }^{33}$, it seems important to compare HL ( $\left.>60 \% \mathrm{MVC}\right)$ and LL (<60\% MVC) RT to MF for ILEX.

Practical treatment approaches using ILEX RT is effective in treating chronic low-back pain ${ }^{32}$. As it seems deconditioning is linked to the development of LBP, ${ }^{34}$ strengthening of this musculature using ILEX RT might be an effective preventative approach. ${ }^{35}$ Furthermore, since strength increases may be related to clinical improvements from ILEX interventions ${ }^{36}$ it seems important to understand the manipulation of RT variables that might optimise strength outcomes. However, to date these loading schemes have not been compared directly, and furthermore, the influence of perceptual responses such as effort and discomfort upon strength outcomes from ILEX exercise has not been considered. It has recently been shown that ILEX RT using LL produces greater acute fatigue and discomfort compared with HL. ${ }^{17}$ As such, when prescribing resistance training for general or clinical populations this might potentiate issues with higher effort exercise (e.g. reaching MF) and result in suboptimal adaptations over a training intervention. Thus, it seems important to examine the effects of loading schemes during ILEX exercise upon these variables.

With the above in mind, the aim of this study was to compare the strength increases of healthy males and females performing ILEX exercise using either HL ( $80 \% \mathrm{MVC})$ or LL (50\% MVC) as well as the perceptual responses of effort and discomfort. 
previous training experience of the isolated lumbar extensors were recruited (see Table 1 for could not lift from the seat. A femur restraint was placed fixed just above the patella and a footboard tightened in driving the participant's shanks upward and back, and thus also the femur. The torque at

of these restraints is to prevent upward movement and rotation, of the pelvis whilst permitting full

\footnotetext{
${ }^{2}$ Inclusion criteria required participants to rate themselves as 'moderately fit' on a customised screening form for physical activity readiness, but not be undertaking structured resistance exercise, or have specific aesthetic or fitness goals (e.g. fat loss, build muscle, compete in any physical contest, event or sport, etc.). Furthermore, all participants were asymptomatic of low back pain and confirmed that they had never undertaken isolated lumbar extension exercise.
} 
139 their heels form the footboard and ensuring the participant could not rotate their pelvis when leaning

140 forwards and backwards (confirmed by an absence of opposite rotation of a rolling pad at the upper

141 posterior pelvis - see pelvic restraint; Figure 1). The head rest was adjusted accordingly whilst

142 participants were informed that all extension force was to be applied to the resistance pad (see Figure

143 1). Participants were assessed to be comfortable and to not be experiencing any pain, numbness, or

144 paraesthesia. Participants were then assessed for lumbar range of motion using a goniometer built in to

145 the ILEX machine (MedX, Ocala, FL, USA), and performed isometric testing using the ILEX machine every

$14612^{\circ}$ beginning at $72^{\circ}$ (full lumbar flexion) through $60^{\circ}, 48^{\circ}, 36^{\circ}, 24^{\circ}, 12^{\circ}$, and $0^{\circ}$ (full lumbar extension) to

147 allow them to experience the technique required and reduce any learning effect. Participants were also

148 familiarised with the rating of perceived effort (RPE-E) ${ }^{3}$ and rating of perceived discomfort (RPE-D) ${ }^{4} 0-10$

149 scales. Participants read the description of anchoring for both scales (detailed elsewhere ${ }^{2,3,11}$ ) and

150 confirmed their understanding of differentiation between effort and discomfort.

The testing protocol for the MedX ILEX machine followed the process described above for the

152

153

154 familiarization session; participants were seated in the ILEX machine and a thigh and femur restraint tightened to permit full lumbar mobility whilst ensuring the pelvis could not rotate. Participants were and $\sim 20 \mathrm{~kg}$ for females, followed by 3 practice isometric actions at full flexion, mid-range of motion, and full extension using 50\% effort. An MVC was then performed at 7 different joint angles (as described above) by the participant gradually building force up to maximal effort over 3 seconds, and then relax their contraction over a further 3 seconds. Participants were provided with verbal encouragement to ensure maximal effort and were permitted $\sim 10$ seconds complete recovery between testing angles. The ILEX machine and restraint system (figure 1) prevents rotation of the pelvis allowing training and testing

\footnotetext{
${ }^{3}$ RPE-E; $0=$ no exertion, $1=$ =xtremely easy, 3=easy, 5=somewhat hard, $7=$ hard, 9=very hard, 10=maximal effort. ${ }^{4}$ RPE-D; $0=$ no discomfort, 1=minor discomfort, 3=mild discomfort, 5=moderate discomfort, 7=severe discomfort, $9=$ very severe discomfort, $10=$ maximal discomfort
} 
161 of the lumbar extensors in isolation. The device has high test-retest reliability values of $r=0.81-0.97$ in

162 asymptomatic persons. ${ }^{37}$ the lumbar extensors for $\sim 60$ seconds using $\sim 27 \mathrm{~kg}$ for males and $\sim 20 \mathrm{~kg}$ for females. This was followed by a single maximal isometric test at $72^{\circ}$ (full lumbar flexion) from which the load equating to $80 \%$ - (HL) or 50\%- (LL) maximum torque was used for a single set of ILEX exercise. The use of this single MVC allowed weekly prescription and progression of exercise training load throughout the intervention. Repetitions were performed taking at least 2 seconds to complete the concentric phase, holding for 1 second in full extension and taking at least 4 seconds for the eccentric phase to ensure standardisation, with visual time feedback on a display in front of the participant. The ILEX machine also provides an audible sound at the completion of each phase of the repetition (e.g. at full flexion $-72^{\circ}$, and full extension $-0^{\circ}$ ) to ensure the full range of motion is performed for each repetition. As fatigue incurred, the repetition duration generally increased but participants were encouraged never to move faster than the predetermined repetition duration. The exercise was ceased when a set endpoint of momentary failure had been achieved as per definition from Steele et al. ${ }^{1}$ i.e. despite their maximum effort, participants could not complete the concentric phase of a repetition. A single set of ILEX exercise was chosen due to the similar strength increases to a multiple-set protocol ${ }^{25}$. Immediately following each training session, each participant was asked to report a value for effort (RPE-E) and discomfort (RPE-D) using the aforementioned 0-10 scales that permitted appropriate differentiation of the 2 perceptions. ${ }^{2,3,11}$ All 

change in strength (considered as both peak MVC and a strength index [SI] calculated as the area under the torque curve across the participants range of motion using the trapezoidal method), and RPE-D, time under load, and number of repetitions performed, averaged across the 6 training sessions. Analysis was not performed for RPE-E since all participants reported maximal effort (i.e. 10) at the cessation of dynamic exercise in both HL and LL. Shapiro-Wilk test was conducted to examine whether data met assumptions variance. Analysis of covariance (ANCOVA) was used for between group comparisons of strength changes (post minus pre values) with baseline measures as covariates. Point estimates for change in SI are provided

further to indicate that significant within group changes occurred if the upper or lower limits do not cross

Portsmouth, Hampshire, UK) and $p<0.05$ set as the limit for statistical significance.

202

203

204

205

206

207

\section{RESULTS}

Between group comparisons using ANCOVA revealed no significant difference for change in $\operatorname{MVC}\left(F_{(1,23)}=0.095, p=0.761\right)$ or SI $\left(F_{(1,23)}=8.514 * 10^{-4}, p=0.977\right)$. Table 2 shows pre-intervention results, post-intervention results, and estimated marginal means for changes in each outcome measure with $95 \% \mathrm{Cls}$ for the changes. All changes were considered to be significant within groups as $95 \% \mathrm{Cls}$ did not cross zero. Figure 2 presents the Gardner-Altman plot with individual responses for change in SI for 
208

209

each group, in addition to the between group difference in change scores with $95 \% \mathrm{Cls}$, and figure 3 shows the same for change in MVC.

Between group comparisons using an independent $t$-test revealed significantly greater time under load and number of repetitions for LL compared with $\mathrm{HL}$ (both $t_{(24)}=-7.865, p<0.001$ ). Between group comparisons using a Mann Whitney-U test for RPE-D revealed significantly greater values for LL compared with $\mathrm{HL}(U=23.500, p=0.002)$. Time under load, number of repetitions, and RPE-D are shown in Table 3

\section{DISCUSSION}

The present study compared low-volume resistance training of the lumbar extensors using heavier- (HL; 80\% MVC) or lighter- (LL; 50\% MVC) load ILEX in asymptomatic, recreationally active males and females. The findings from this study serve to support the efficacy of both HL and LL ILEX exercise towards strengthening of the lumbar extensors using a low volume (single-set), low frequency (1x/week) approach.

The present data suggests that there are significant increases in ILEX strength (for both MVC and SI) for HL and LL resistance training, with no between group differences. This is supportive of previous research which suggests that, where tested by impartial means, strength increases are similar between heavier- and lighter-load resistance training when taken to the point of momentary failure. ${ }^{2,3,4}$ This similarity of strength increases is hypothesized to result from the maximal synchronous (in the case of $\mathrm{HL}$ ), or sequential (in the case of $\mathrm{LL}$ when exercise is continued to MF), motor unit recruitment, ${ }^{2}$ as per the size principle. 5,6 The impartial methods used herein are important for strength training practitioners to consider since they serve to minimise skill acquisition and the resultant strength improvements through the practicing of lifting heavy loads. In context, people are likely to seek improvement in ILEX strength for the purpose of reducing the likelihood or severity of chronic low-back pain, and as such we 
231 believe that the methods used herein (e.g. dynamic training and isometric testing) are essential for

232 avoiding the confounding effects of skill acquisition in determining whether general strength gains have

233 occurred. Indeed, previous work has actually shown that ILEX strength is associated with lifting

234 capacity ${ }^{39,40}$ which improves after ILEX resistance training, ${ }^{41}$ as does the amount of resistance moved in

235 the deadlift exercise. ${ }^{24}$ Other studies also suggest that ILEX strength may be related to balance and

236 motor control during gait. ${ }^{42,43}$ Furthermore, isometric strength increases following dynamic ILEX training

237 are shown to result in a reduction in chronic low-back pain at both heavier-loads $\left(80 \% \mathrm{MVC} \mathrm{C}^{26-28}\right)$, and

238 lighter-loads (35-50\% MVC $29,30,44)$.

As per previous research comparing $\mathrm{HL}$ and $\mathrm{LL}$ resistance training to momentary failure, ${ }^{3}$

participants in the LL group performed a significantly greater number of repetitions (LL $=26 \pm 8 \mathrm{vs} . \mathrm{HL}=8$

\pm 3 repetitions; $p<0.001$ ) resulting in a significantly longer time-under-load ( $L L=158.5 \pm 47.0$ vs. $\mathrm{HL}=50.5$

\pm 15.4 seconds; $p<0.001$ ) compared to the HL group. Maximal effort was confirmed by the use of an RPE-

E scale where all participants in both $\mathrm{HL}$ and $\mathrm{LL}$ conditions reported maximal values (e.g. 10). Previous

research has reported counterintuitive (e.g. submaximal) values for effort measured by traditional RPE

scales when participants have performed different exercises at different relative loads to the point of

momentary failure. ${ }^{45}$ As discussed, it seems likely that previously most trainees anchored their

perceptions of effort upon their perceptions of discomfort. ${ }^{11}$ However, the present study adds to the

body of literature suggesting that when instructed, participants can differentiate their perceptions of

effort and discomfort. ${ }^{3,16,17}$ The present data supports this previous research; that longer time-under-

load results in a greater degree of discomfort, as reported by the participants in the LL compared to the

$\mathrm{HL}$ condition ( $\mathrm{LL}=7.8$ vs. $\mathrm{HL}=4.8, p=0.002)$.

Within the present study we did not measure metabolite accumulation or blood-based markers

for metabolic stress, however, we have previously hypothesised that the greater values for discomfort resulting from a longer time-under-load are likely a product of a decrease in $\mathrm{pH}$, elevated blood lactate 
255 (BLa), cortisol, and inorganic phosphate $\left(\mathrm{P}_{\mathrm{i}}\right)$ along with increases in $\mathrm{H}^{+}$as a result of the prolonged

256 elevated adenosine triphosphate (ATP) production. ${ }^{18-21}$ Behm, et al. ${ }^{46}$ suggested that following LL

257 resistance exercise (20RM) fatigue occurred as a result of peripheral factors, potentially a decrease in

258 the contractile strength of muscle fibres and an inability to transmit the impulse across the

259 neuromuscular junction. ${ }^{47,48}$ Perceived effort is likely centrally mediated thus explaining similar results

260 for HL and LL conditions in the present study, whereas perceptions of discomfort may be more closely

261 associated with afferent feedback. ${ }^{9}$

262 Of course, the present study is not without its limitations. Notably, whilst we have considered

263 strength increases and perceptual responses to HL and LL ILEX exercise, these results in an

264 asymptomatic participant sample might not be applicable to those with non-specific mechanical chronic

265 low-back pain. Symptomatic persons might be predisposed to a greater degree of discomfort through

266 rehabilitation as a result of their low-back pain for both $\mathrm{HL}$ and $\mathrm{LL}$ exercise. As such future research

267 might consider a similar research design conducted with symptomatic chronic low-back pain patients

268 and compare the perceptual responses between asymptomatic and symptomatic persons, as well as

269 strength and pain reduction improvements. In addition, whilst the use of a regular MVC was appropriate

270 to ensure progressive and specific loading strategies, it might have presented a confounding issue. Since

271 both $\mathrm{HL}$ and $\mathrm{LL}$ training groups performed a single MVC at $72^{\circ}$ (full flexion) each week, this practice of

272 the test by both groups might have converged strength increases between groups. However, this would

273 likely impact the MVC to the greatest degree with potentially minimal impact on the SI data (which was

274 a product of all tested angles). Further research might also consider the longevity of the exercise

275 intervention since previous research has suggested that repeated exposure to exercise conditions

276 known to cause discomfort increases pain tolerance. ${ }^{49}$

277 Finally, we should discuss the sample size of participants. We did not conduct an a-priori power

278 analysis but rather collected data throughout the duration of availability of a research assistant. 
279 However, post-hoc we have calculated the Cohen's $f$ effect size between two intervention groups in a

280 prior study from our lab ${ }^{25}$ as 0.16 - a small effect. Assuming that a moderate effect might be considered

281 meaningful here, G*Power suggests with an ANCOVA model as used herein, that $n=128$ would be

282 required for power of 0.8 at an alpha of 0.05 to detect an effect of 0.25 . As many might perhaps agree,

283 this is an unrealistic number for a study in this field to recruit. As such we should consider that the

284 present study might be underpowered, although from the very similar point estimates and precision of

285 those estimates it seems that any effect, if one were to truly exist, would be very small and practically

286 difficult to justify in terms of meaningfulness.

\section{Conclusion}

The present study is the first to compare HL (80\% MVC) and LL (50\% MVC) resistance training for the lumbar extensors in recreationally active males and females. Our data supports previous research that $\mathrm{HL}$ and $\mathrm{LL}$ resistance training produce similar chronic strength adaptations. With the above in mind, it is important to consider that a historical approach to training the muscles of the trunk has been to use lighter loads and higher repetition ranges. This is likely because of the predominance of type I muscle fibres $^{23}$, and the resulting repeated lower force actions needed for postural control and stability.

However, based on the evidence presented herein as well as previous literature ${ }^{3,16,17}$ this lighter-load exercise likely produces a greater degree of discomfort which might result in persons failing to perform higher effort exercise (e.g. reaching MF) in real world settings, and could result in suboptimal adaptations over a training intervention. This is particularly important since poor lumbar strength is associated with low back pain. The present data also adds to the body of literature that low-volume (single-set), and low frequency (1 day/week) ILEX exercise performed to momentary failure produces significant strength increases. As such, we suggest that this minimal dose approach to ILEX exercise might be manageable for persons to increase their ILEX strength with the aim of reducing risk of low back injury and pain. ${ }^{35}$ 
304

305

306

307

308

309

310

311

312

313

314

315

316

317

318

319

320

321

322

323

324

325

326

327

328

329

330

331

332

\section{REFERENCES}

1. Steele J, Fisher J, Giessing J, Gentil P. Clarity in reporting terminology and definitions of set endpoints in resistance training. Muscle Nerve 2017;56(3):368-374.

2. Fisher J, Steele J, Smith D. High- and Low-Load Resistance Training: Interpretation and Practical Application of Current Research Findings. Sport Med 2017;47(3):393-400.

3. Fisher JP, Ironside M, Steele J. Heavier- and lighter-load resistance training to momentary failure produce similar increases in strength with differing degrees of discomfort. Muscle Nerve 2017;56(4):797-803.

4. Schoenfeld BJ, Grgic J, Ogborn D, Krieger JW. Strength and hypertrophy adaptations between low- versus high-load resistance training: a systematic review and meta-analysis. J Strength Cond Res 2017;31(12):3508-3523.

5. Denny-Brown D, Pennybacker JB. Fibrillation and fasciculation in voluntary muscle. Brain 1938;61:311-312.

6. Potvin JR, Fuglevand AJ. A motor unit-based model of muscle fatigue. PLoS Comput Biol 2017;13(6):e1005581.

7. Schoenfeld BJ, Wilson JM, Lowery RP, Krieger JW. Muscular adaptations in low-versus high-load resistance training: A meta-analysis. Eur J Sport Sci 2014;20:1-10.

8. Abbiss CR, Peiffer JJ, Meeusen R, Skorski S. Role of ratings of perceived exertion during selfpaced exercise: what are we actually measuring? Sports Med 2015;45:1235-1243.

9. Marcora S. Perception of effort during exercise is independent of afferent feedback from skeletal muscles, heart, and lungs. J Appl Physiol 2009;106:2060-2062.

10. Smirnaul Bde P. Sense of effort and other unpleasant sensations during exercise: clarifying concepts and mechanisms. Br J Sports Med 2012;46: 308-311.

11. Steele J, Fisher J, McKinnon S, McKinnon P. Differentiation between perceived effort and discomfort during resistance training in older adults: Reliability of trainee ratings of effort and discomfort, and reliability and validity of trainer ratings of trainee effort. J Trainol 2017;6(1):1-8.

12. Giessing J, Fisher J, Steele J, Rothe F, Raubold K, Eichmann B. The effects of low-volume resistance training with and without advanced techniques in trained participants. J Sports Med Phys Fitness 2016;56(3):249-258. 
333

13. Giessing J, Eichmann B, Steele J, Fisher J. A comparison of low volume 'high-intensity-training' and high volume traditional resistance training methods on muscular performance, body composition, and subjective assessments of training. Biol Sport 2016;33(3):241-249.

14. Steele J, Endres A, Fisher J, Gentil P, Giessing J. Ability to predict repetitions to momentary failure is not perfectly accurate, though improves with resistance training experience. PeerJ 2017c;5:e4105.

15. Lemos EA, Caldas LC, Leopoldo APL, Leopoldo AS, Ferreira LG, Lunz W. The perception of effort is not a valid tool for establishing the strength-training zone. J Human Sport Exerc 2017;12(3):593606

16. Fisher JP, Farrow J, Steele J. Acute fatigue, and perceptual responses to resistance exercise. Muscle Nerve 2017;56:E141-E146.

17. Stuart C, Steele J., Gentil P, Giessing J, Fisher JP. Fatigue and perceptual responses of heavierand lighter-load isolated lumbar extension resistance exercise in males and females. PeerJ 2018;6:e4523.

18. Genner KM, Weston M. A comparison of workload quantification methods in relation to physiological responses to resistance exercise. J Strength Cond Res 2014;28(9):2621-2627.

19. Schott J, McCully K, Rutherford OM. The role of metabolites in strength training II. Short versus long isometric contractions. Eur J Appl Physiol 1995;71:337-341.

20. Takada S, Okita K, Suga T, Omokawa M, Kadoguchi T, Sato T, Takahashi M, Yokota T, Hirabayashi K, Morita N, Horiuchi M, Kinugawa S, Tsutsui H. Low-intensity exercise can increase muscle mass and strength proportionally to enhance metabolic stress under ischemic conditions. J Appl Physiol 2012;113:199-205.

21. MacDougall JD, Ray S, Sale DG, McCartney N, Lee P, Garner S. Muscle substrate utilization and lactate production during weight lifting. Can J Appl Physiol 1999;24:209-215.

22. de Morree HM, Klein C, Marcora SM. Perception of effort reflects central motor command during movement execution. Psychophysiology 2012;49(9):1242-1253.

23. Thorstensson A, Carlson H. Fibre types in human lumbar back muscles. Acta Physiol Scand 1987;131(2):195-202.

24. Fisher J, Bruce-Low S, Smith D. A randomized trial to consider the effect of Romanian deadlift exercise on the development of lumbar extension strength. Phys Ther Sport 2013;14(3):139-145.

25. Steele J, Fitzpatrick A, Bruce-Low S, Fisher J. The effects of set volume during isolated lumbar extension resistance training in recreationally trained males. PeerJ 2015;3:e878. 
365

26. Bruce-Low S, Smith D, Burnet S, Fisher J, Bissell G, Webster L. One lumbar extension training session per week is sufficient for strength gains and reductions in pain in patients with chronic low back pain ergonomics. Ergonomics 2012;55(4); 500-507.

27. Steele J, Bruce-Low S, Smith D, Jessop D, Osborne N. A randomized controlled trial of limited range of motion lumbar extension exercise in chronic low back pain. Spine 2013;38(15):12451252.

28. Steele J, Bruce-Low S, Smith D, Jessop D, Osborne N. Isolated lumbar extension resistance training improves strength, pain, and disability, but not spinal height or shrinkage ("Creep") in participants with chronic low back pain. Cartilage E-Pub ahead of print. doi.org/10.1177/1947603517695614.

29. Helmhout PH, Harts CC, Staal JB, Candel MJJM, de Bie RA. Comparison of a high-intensity and a low-intensity lumbar extensor training program as a minimal intervention treatment in low back pain: a randomized trial. Eur Spine J 2004;13(6):537-547.

30. Harts CC, Helmhout PH, de Bie RA, Staal JB. A high-intensity lumbar extensor strengthening program is little better than a low-intensity program or a waiting list control group for chronic low back pain: a randomised clinical trial. Aust J Physiother 2008;54(1):23-31.

31. Dankel S, Jessee M, Mattocks K, Mouser JG, Counts BR, Buckner SL, Loenneke JP. Training to Fatigue: The Answer for Standardization When Assessing Muscle Hypertrophy? Sports Med 2016;47(6): 1021-1027.

32. Steele J, Bruce-Low S, Smith D. A review of the clinical value of isolated lumbar extension resistance training for chronic low back pain. Phys Med Rehab 2015;7(2):169-187.

33. Gentil P, Fisher J, Steele J. A Review of the Acute Effects and Long-Term Adaptations of Single- and Multi-Joint Exercises during Resistance Training. Sports Med 2017;47(5):843-855.

34. Steele J, Bruce-Low S, Smith D. A reappraisal of the deconditioning hypothesis in low back pain: review of evidence from a triumvirate of research methods on specific lumbar extensor deconditioning. Curr Med Res Opin 2014;30(5):865-911.

35. Steele J, Bruce-Low S, Smith D. A review of the specificity of exercises designed for conditioning the lumbar extensors. Br J Sports Med 2015;49(5):291-297.

36. Steele J, Fisher J, Perrin C, Conway R, Bruce-Low S, Smith D. Does change in isolated lumbar extensor muscle function correlate with good clinical outcome? A secondary analysis of data on change in isolated lumbar extension strength, pain, and disability in chronic low back pain. Disabil Rehabil 2018;12:1-9 
397

398

399

400

401

402

403

404

405

406

407

408

409

410

411

412

413

414

415

416

417

418

419

420

421

422

423

424

425

426

427

428
37. Graves JE, Pollock ML, Carpenter DM, Leggett SH, Jones A, MacMillan M, Fulton M. Quantitative assessment of full range of motion isometric Lumbar Extension Strength. Spine 1990;15:504509.

38. Ho, J., \& Claridge-Chang, A. (2017). "Estimation Statistics" website http://www.estimationstats.com, accessed on [13th June 2018]

39. Reyna JR, jr, Leggett SH, Kenney K, Holmes B, Mooney V. The effect of lumbar belts on isolated lumbar muscle. Strength and dynamic capacity. Spine 1995;20:68-73.

40. Matheson LN, Leggett S, Mooney V, Schneider K, Mayer J. The contribution of aerobic fitness and back strength to lift capacity. Spine 2002;27:1208-1212.

41. Mooney V, Matheson L, Holmes D, Leggett S, Grant J, Negri S, Holmes B. Effect of focused strength training after low back injury. Paper presented at: North American Spine Society Annual Meeting; 1993; San Diego, CA.

42. Steele J, Bruce-Low S, Smith D, Jessop D, Osborn N. Lumbar kinematic variability during gait in chronic low back pain and associations with pain, disability and isolated lumbar extension strength. Clin Biomech 2014;29:1131-1138.

43. Behennah J, Conway R, Fisher J, Osborne N, Steele J. The relationship between balance performance, lumbar extension strength, trunk extension endurance, and pain in participants with chronic low back pain, and those without. Clin Biomech 2018;53:23-30.

44. Risch SV, Norvell NK, Pollock ML, Risch ED, Langer H, Fulton M, Graves JE, Leggett SH. Lumbar strengthening in Chronic low back pain patients physiological and psychological benefits. Spine 1993;18(2):232-239.

45. Shimano T, Kraemer WJ, Spiering BA, Volek JS, Hatfield DL, Silvestre R, Vingren JL, Fragala MS, Maresh CM, Fleck SJ, Newton RU, Spreuwenberg LP, Häkkinen K. Relationship between the number of repetitions and selected percentages of one repetition maximum in free weight exercises in trained and untrained men. J Strength Cond Res 2006;20:819-823.

46. Behm DG, Reardon G, Fitzgerald J, Drinkwater E. The effect of 5, 10, and 20 repetitions maximums on the recovery of voluntary and evoked contractile properties. J Strength Cond Res 2002;16:209-218.

47. Boyas S, Guevel A. Neuromuscular fatigue in healthy muscle: underlying factors and adaptation mechanisms. Ann Phys Rehabil Med 2011;54:88-108.

48. Gandevia SC. Spinal and supraspinal factors in human muscle fatigue. Physiol Rev 2001;81:17251789. 
49. O'Leary TJ, Collett J, Howells K, Morris MG. High but not moderate-intensity endurance training increases pain tolerance: a randomised trial. Eur J Appl Physiol 2017;117(11):2201-2210.

431 
Table $\mathbf{1}$ (on next page)

Participant characteristics 
1 Table 1. Participant characteristics

\begin{tabular}{lcc}
\hline Variable & $\mathrm{HL}(n=13)$ & $\mathrm{LL}(n=13)$ \\
\hline Age (years) & $23.1 \pm 5$ & $22.1 \pm 2$ \\
Stature $(\mathrm{cm})$ & $172.3 \pm 9.8$ & $175.3 \pm 6.3$ \\
Body mass $(\mathrm{kg})$ & $71.0 \pm 13.1$ & $72.8 \pm 9.5$
\end{tabular}

$2 \quad \mathrm{HL}=$ Heavier load (80\% MVC), LL=lighter load (50\%MVC), MVC=maximal voluntary contraction

3 
Table 2 (on next page)

Pre-intervention results, post-intervention results, and changes in strength 
1 Table 2. Pre-intervention results, post-intervention results, and changes in strength

\begin{tabular}{|c|c|c|c|}
\hline Variable & 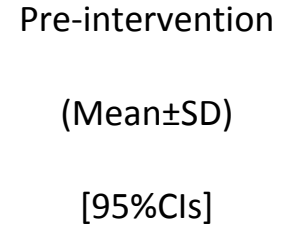 & $\begin{array}{l}\text { Post-intervention } \\
\qquad \begin{array}{c}\text { (Mean } \pm \text { SD) } \\
{[95 \% \mathrm{Cls}]}\end{array}\end{array}$ & $\begin{array}{c}\text { Changes (Estimated } \\
\text { Marginal Means } \\
[95 \% \mathrm{Cls}])\end{array}$ \\
\hline \multicolumn{4}{|c|}{ SI (Nm·degrees) } \\
\hline$H L$ & $\begin{array}{c}19407 \pm 5395 \\
\text { [16475 to } 22339]\end{array}$ & $\begin{array}{c}22300 \pm 5665 \\
\text { [19221 to } 25379]\end{array}$ & 2891 [1612 to 4169 ] \\
\hline LL & $\begin{array}{c}18930 \pm 4245 \\
\text { [16623 to } 21237]\end{array}$ & $\begin{array}{c}21790 \pm 5165 \\
\text { [18981 to } 24599]\end{array}$ & 2865 [1587 to 4144$]$ \\
\hline \multicolumn{4}{|l|}{$\mathrm{MVC}(\mathrm{Nm})$} \\
\hline $\mathrm{HL}$ & $\begin{array}{l}344.3 \pm 110.16 \\
\text { [284.4 to } 404.2 \text { ] }\end{array}$ & $\begin{array}{c}395.1 \pm 108.6 \\
\text { [336.1 to } 454.1]\end{array}$ & 51.7 [24.4 to 79.1$]$ \\
\hline LL & $\begin{array}{c}326.2 \pm 66.26 \\
{[290.2 \text { to } 362.2]}\end{array}$ & $\begin{array}{c}373.2 \pm 74.77 \\
{[332.5 \text { to } 413.9]}\end{array}$ & 46.0 [18.6 to 73.3$]$ \\
\hline
\end{tabular}

2 SI=strength index, HL=Heavier load (80\% MVC), LL=lighter load (50\% MVC), MVC=maximal voluntary

3 contraction, $\mathrm{SE}=$ standard error, $\mathrm{Cls}=$ confidence intervals, $\mathrm{Nm}=$ Newton metres 


\section{Table 3(on next page)}

Time under load, number of repetitions performed, and RPE-D

Note: RPE-D is presented as median and interquartile range 
1 Table 3. Time under load, number of repetitions performed, and RPE-D

\begin{tabular}{lcc}
\hline Variable & $\mathrm{HL}(n=13)$ & $\mathrm{LL}(n=13)$ \\
\hline Time under load (seconds) & $50.5 \pm 15.4$ & $158.5 \pm 47.0$ \\
Number of repetitions $(n)$ & $8 \pm 3$ & $26 \pm 8$ \\
RPE-D $(0 \text { to } 10)^{*}$ & $4.8 \pm 2.5$ & $7.8 \pm 1.8$
\end{tabular}

2 Note: RPE-D is presented as median and interquartile range

$3 \mathrm{HL}=$ heavier load $(80 \% \mathrm{MVC})$, LL=lighter load $(50 \% \mathrm{MVC}), \mathrm{MVC}=$ maximal voluntary contraction, RPE-D= 4 rating of perceived discomfort

5

6

7

8 
Figure 1

Restraint system for the MedX Isolated lumbar extension machine

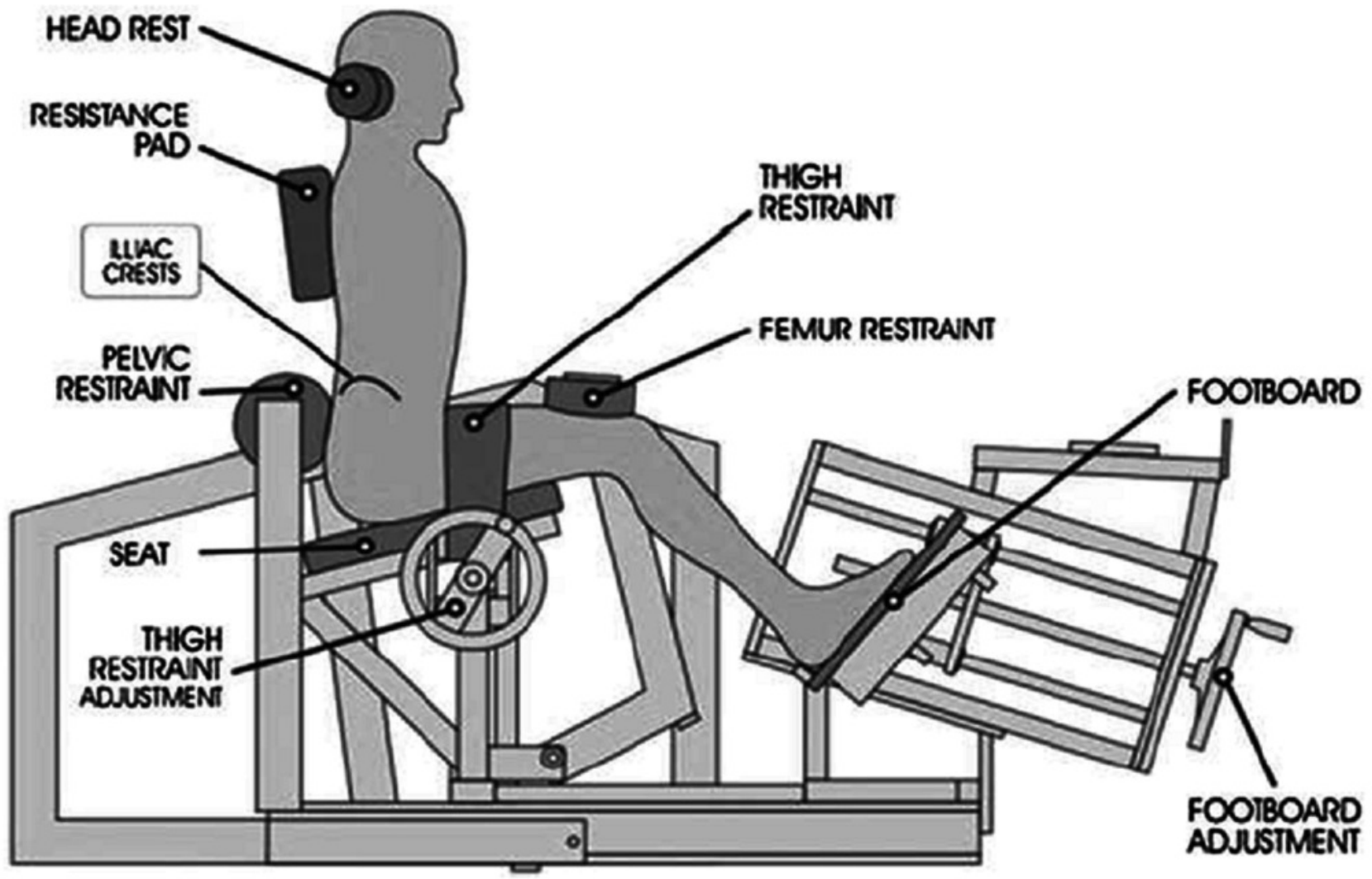


Figure 2

Gardner-Altman plot of change in SI
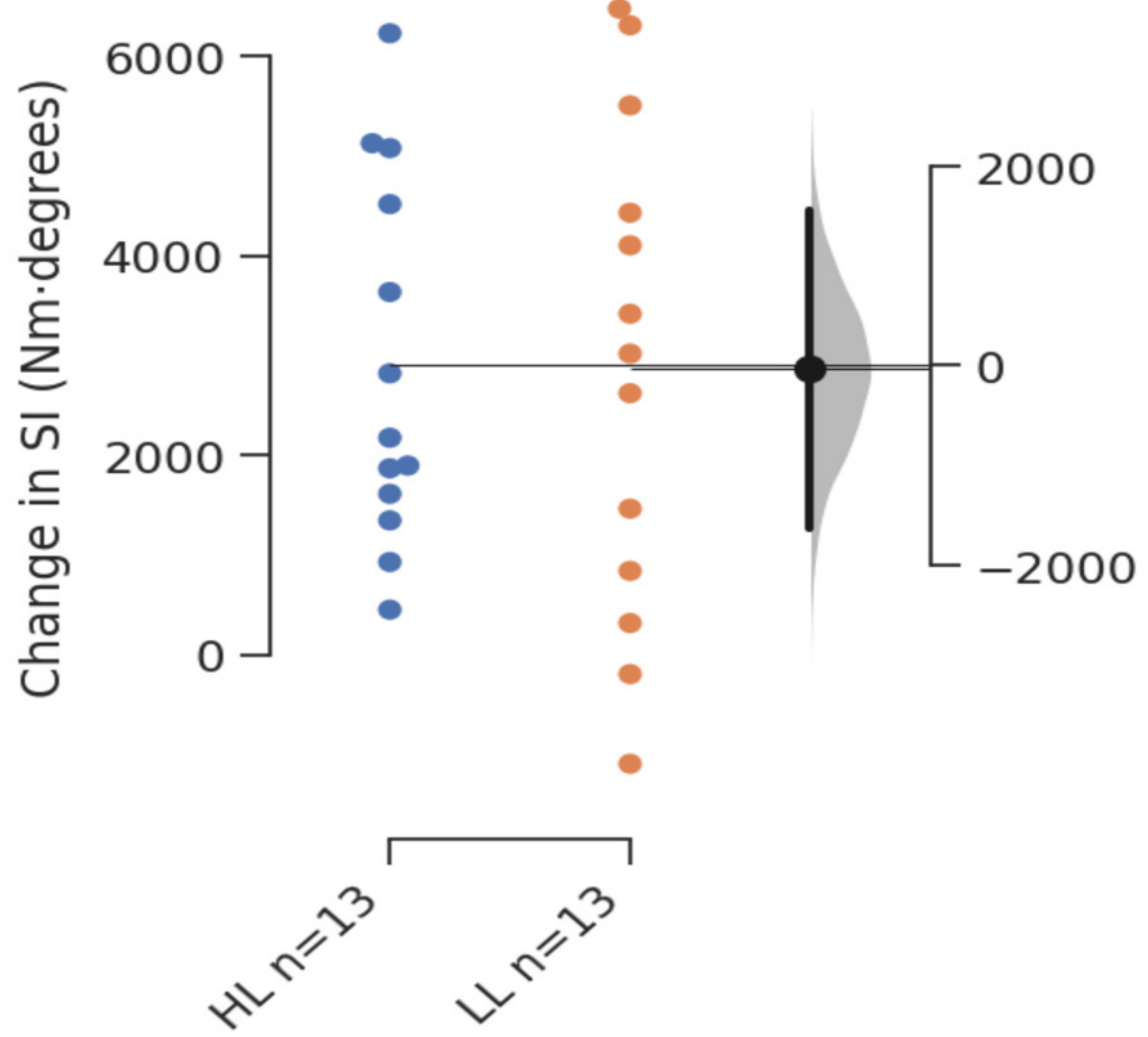
Figure 3

Gardner-Altman plot of change in MVC

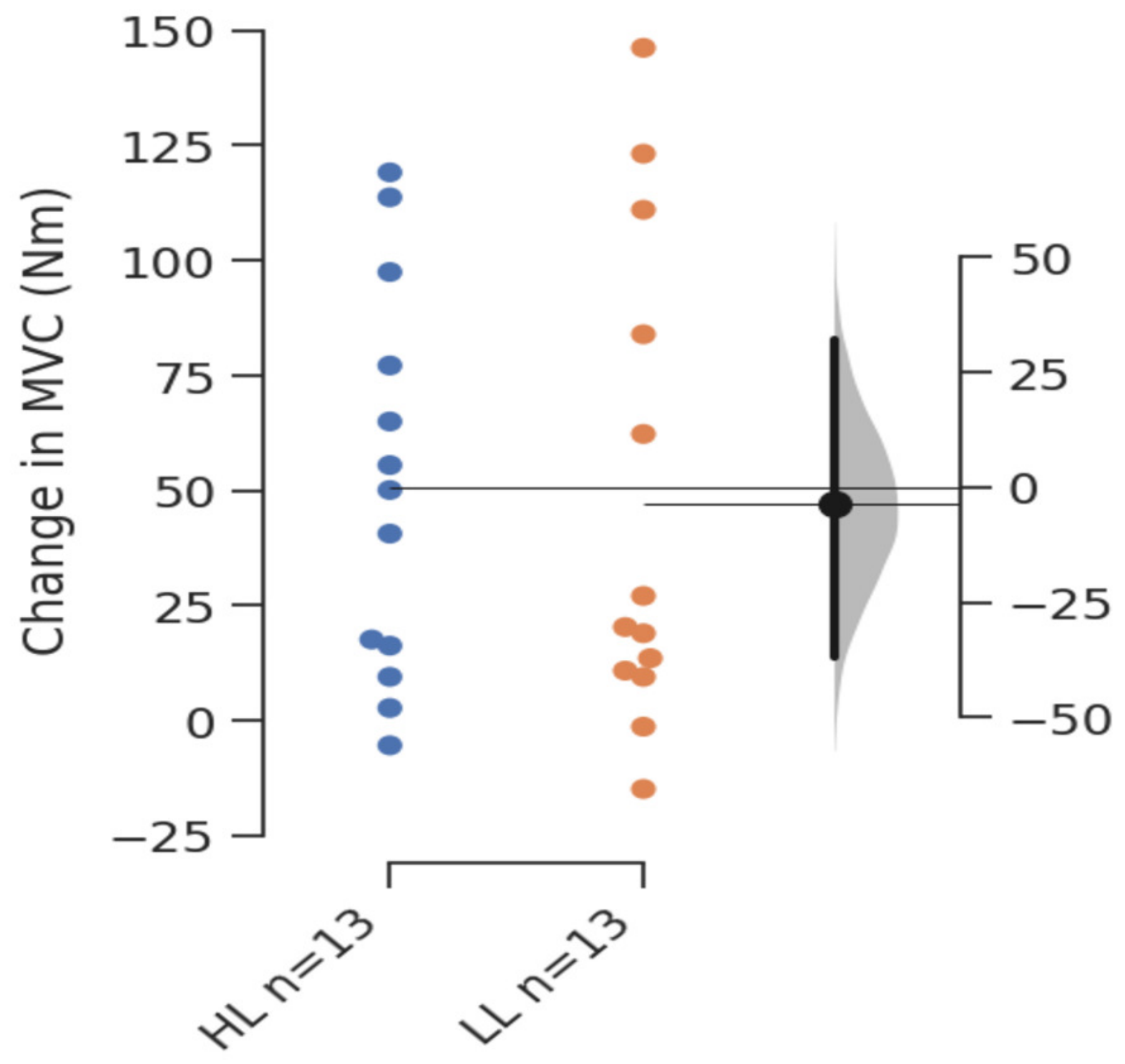

